\title{
Tiefe Venenthrombose: Zeitkritische Wiederherstellung des Blutflusses
}

Li W et al. Time-Restricted Salutary Effects of Blood Flow Restoration on Venous Thrombosis and Vein Wall Injury in Mouse and Human Subjects. Circulation 2021; doi:10.1161/CIRCULATIONAHA.120.049096

Bis zu $50 \%$ der Patienten mit proximaler tiefer Venenthrombose entwickeln ein postthrombotisches Syndrom (PTS). Frühere Studien zeigten nur einen begrenzten klinischen Nutzen einer Katheter-geführten Thrombektomie zur Reduzierung des PTS. In der vorliegenden Studie untersuchten die Autoren einen möglichen zeitabhängigen Effekt Katheter-geführter Therapien auf die Auflösung einer tiefen Venenthrombose und die Entwicklung eines PTS.

Die Wiederherstellung des Blutflusses (restoration of blood flow, RBF) unterstützt die Auflösung der tiefen Venenthrombose (deep venous thrombosis, DVT), und eine Katheter-gestützte pharmakomechanische Thrombolyse (pharmacomechanical catheter-directed thrombolysis, PCDT) kann die klinischen Ergebnisse verbessern.

Die Forscher erzeugten eine DVT in einem Mausmodell durch vollständige Ligatur der V. cava inferior, gefolgt von einer mechanischen Aufhebung der Ligatur, um die Wiederherstellung des Blutflusses zu fördern. Untergruppen der Mäuse erhielten außerdem 2 Tage nach Aufhebung der Ligatur einen rekombinanten Plasminogen- aktivator (rtPA), um die Auswirkungen des rtPA auf okklusive versus nichtokklusive DVT zu analysieren. Außerdem führten die Forscher eine exploratorische Posthoc-Analyse der ATTRACT-PCDT-Studie durch, in der sie die Effekte spezifischer Zeitfenster für die PCDT auf die Lebensqualität und den Schweregrad des PTS bestimmten. Die Autoren definierten eine frühe RBF als RBF bis Tag 4 und eine späte RBF, wenn diese erst nach Tag 4 festgestellt werden konnte.

In Mäusen, in denen die Ligatur der V. cava inferior entfernt wurde, kam es zu einer schnelleren und vollständigeren RBF-Rate verglichen zu Mäusen, bei denen lediglich eine Scheinintervention zur Entfernung der Ligatur erfolgte. In der scheinbehandelten Kontrollgruppe zeigten einige Mäuse ebenfalls eine Reperfusion durch neu gebildete kleine Venen um die Ligatur.

Nach der Aufhebung der Ligatur an Tag 2 zeigte die frühe RBF-Subgruppe (RBF bis Tag 4) eine Reduktion von $\sim 30 \%$ in der Thrombusmasse an Tag 8. Die späte RBFSubgruppe (RBF zwischen Tag 4 und 8) wies hingegen keine signifikante Reduktion der Thrombusparameter im Vergleich zu Mäusen ohne RBF auf.
Exogen verabreichtes rtPA an Tag 4 in Mäusen ohne RBF reduzierte die Thrombuslast bis Tag 8 signifikant. In Mäusen mit früher RBF bis Tag 4 zeigte die Verabreichung des rtPA jedoch keinen Einfluss auf die Thrombuslast an Tag 8. Insgesamt scheinen die Vorteile einer frühen rtPAGabe zur Reduzierung der Thrombuslast und Venenwandschädigung auf okklusive Thromben beschränkt zu sein.

In Mäusen mit einer frühen RBF bis Tag 4 zeigten sich signifikant reduzierte mRNALevel für Entzündungsmediatoren und Mediatoren für Synthese und Abbau der extrazellulären Matrix. Histologische Untersuchungen von Mäusen mit früher RBF wiesen eine signifikante Reduktion in der Venenwand von F4/80+-Makrophagen und FSP1 +-Fibroblasten auf.

Die Autoren teilten Patienten aus der ATTRACT-Studie basierend auf der Zeit vom Auftreten der Symptome bis zur Randomisierung (symptom-onset-to-randomization, SOR) in 3 Subgruppen ein. Für Patienten in der SOR-4-9-Tage-Subgruppe war eine PCDT mit einer signifikant größeren Verbesserung im VEINES-QoL (Venous Insufficiency Epidemiological and Economic Study Quality-of-Life) bei allen Nachbeobach- 
tungsbesuchen verbunden. Der größte Vorteil der PCDT auf den VEINES-QoL zeigte sich in der SOR-Tag-4-8-Subgruppe. Die PCDT verbesserte die VEINES-QoL-Ergebnisse jedoch nicht in den sehr frühen SORGruppen (<4 Tage) oder in der späten SOR-Gruppe (>8 Tage).

\section{FAZIT}

Die mechanische und pharmakologische Wiederherstellung des Blutflusses bei Venenthrombose ist zeitkritisch. Eine frühere, zielgerichtete Wiederherstellung des Blutflusses reduziert die Thrombuslast und die Schädigung der Venenwand im Maus- modell und hat das Potenzial, die Lebensqualität in Patienten mit tiefer Venenthrombose zu verbessern und die Schwere eines postthrombotischen Syndroms zu verringern.

Dr. Bettina Heberer, Herxheim am Berg 